\title{
TETRAGONAL OR HEXAGONAL SYMMETRY IN MODELING OF FAILURE CRITERIA FOR TRANSVERSELY ISOTROPIC MATERIALS
}

\author{
Artur GANCZARSKI*, Michał ADAMSKI \\ *Institute of Applied Mechanics, Department Mechanical Engineering, \\ Cracow University of Technology, 31-864 Kraków al. Jana Pawła II 37, Poland \\ artur.ganczarski@pk.edu.pl, michal.a.krakow@gmail.com
}

\begin{abstract}
Present work deals with modeling of failure criteria for transversely isotropic materials. Analysis comprises two classes of symmetry: Tsai-Wu tetragonal and new Tsai-Wu based hexagonal. Detail analysis of both classes of symmetry with respect to their advantages as well as limitations is presented. Finally, simple comparison of differences between limit curves corresponding to cross sections by planes of transverse isotropy, orthotropy and shear plane is done.
\end{abstract}

Key words: Transverse Isotropy, Tetragonal and Hexagonal Symmetry, Tsai-Wu Criterion

\section{INTRODUCTION}

Formulation of the initial yield or failure criteria for modern materials has to consider material anisotropy, tension-compression asymmetry or hydrostatic pressure sensitivity. In general, there exist two competitive but complementary schools to capture the above behaviours. The first explicit approach is based on a direct concept of common invariants of stress and anisotropy tensors (Sayir, 1970; Goldenblat and Kopnov, 1966; Tsai and Wu, 1971; Murakami, 2012; etc.) The second implicit approach is based on a direct extension of the isotropic-type yield/failure criteria to capture anisotropic behaviour as well as strength differential effect and pressure sensitivity by the use of linear transformation tensors for single stress invariants (Khan et al., 2007; Cazacu and Barlat, 2004 and others). Although the common invariants-based formulation is more mathematically rigorous, but complicated by the use of the second-, fourth-rank structural tensors, a direct extension of classical isotropic criteria to anisotropy is very efficient and broadly examined (Ganczarski and Skrzypek, 2014).

Basic result of the present paper is to propose the new orthotropic criterion of failure initiation, being the extended Tsai-Wu type equation. This new criterion is capable of capturing orthotropic limit surface description, without ellipticity loss even for arbitrarily high orthotropy degree. This is by contrast to the classical Tsai-Wu (1971) criterion (eg. Ottosen and Ristinmaa, 2005), where for high orthotropy degree inadmissible degeneration of the limit surface may occur. This enhanced Tsai-Wu's type limit surface is represented by the elliptic paraboloid, the axis of which is different from hydrostatic axis in the space of principal stresses, hence the criterion no longer satisfies the property of deviatoricity.

The other important feature of the proposed criterion is concerned with the particular case of transversely isotropic hexagonal symmetry, for which it is possible to achieve coincidence with the same proportion of isotropic Huber-von Mises-Hencky condition in the transverse isotropy plane, which may occur beneficial when compared to the deviatoric Tsai-Wu formulation for which above reducibility does not hold.

\section{TRANSVERSELY ISOTROPIC TSAI-WU FAILURE CRITERION}

In a general case of brittle materials that exhibit anisotropy (e.g. concrete, ceramic materials, rocks, composite materials, etc.) and tension-compression asymmetry, a transversely isotropic Tsai-Wu criterion of initial failure (Tsai and Wu, 1971) is applicable:

$$
\begin{gathered}
F\left[\left(\sigma_{y}-\sigma_{z}\right)^{2}+\left(\sigma_{z}-\sigma_{x}\right)^{2}\right]+H\left(\sigma_{x}-\sigma_{y}\right)^{2}+2 L \tau_{x y}^{2} \\
+2 M\left(\tau_{z x}^{2}+\tau_{z y}^{2}\right)+P\left(\sigma_{x}+\sigma_{y}\right)+Q \sigma_{z}=1
\end{gathered}
$$

Eq. (1) contains only 5 independent material coefficients referring to appropriate tensile and compressive strengths $\mathrm{k}_{\mathrm{tx}}, \mathrm{k}_{\mathrm{cx}}$, $\mathrm{k}_{\mathrm{tz}}, \mathrm{k}_{\mathrm{cz}}$ and shear strength $\mathrm{k}_{\mathrm{zx}}$, hence, in order to calibrate it the following tests must be performed:

$$
\begin{array}{lll}
\sigma_{x}=k_{t x} & \rightarrow & (F+H) k_{t x}^{2}+P k_{t x}=1 \\
\sigma_{x}=-k_{c x} & \rightarrow & (F+H) k_{c x}^{2}-P k_{c x}=1 \\
\sigma_{z}=k_{t z} & \rightarrow & 2 F k_{t z}^{2}+Q k_{t z}=1 \\
\sigma_{z}=-k_{c z} & \rightarrow & 2 F k_{c x}^{2}-Q k_{c z}=1 \\
\tau_{z x}=k_{z x} & \rightarrow & 2 M k_{z x}^{2}=1
\end{array}
$$
form:

Solution of Eqs. (2) with respect to F, H, M, P and Q takes the

$$
\begin{gathered}
F=\frac{1}{2 k_{t z} k_{c z}} \quad H=\frac{1}{k_{t x} k_{c x}}-\frac{1}{2 k_{t z} k_{c z}} \quad M=\frac{1}{2 k_{z x}^{2}} \\
P=\frac{1}{k_{t x}}-\frac{1}{k_{c x}} \quad Q=\frac{1}{k_{t z}}-\frac{1}{k_{c z}}
\end{gathered}
$$

Magnitude of material coefficient $L$, referring to shear strength in plane of transverse isotropy, is not independent and can be calculated from the following known relation (see Chen and Han, 1995 and Ganczarski and Skrzypek, 2013):

$2 L=2(F+2 H)=\frac{4}{k_{t x} k_{c x}}-\frac{1}{k_{t z} k_{c z}}$

Hence, after substitution of Eqs. (3)-(4) to Eq. (1) one can get 
final form of the transversely isotropic Tsai-Wu criterion:

$$
\begin{gathered}
\frac{\sigma_{x}^{2}+\sigma_{y}^{2}}{k_{t x} k_{c x}}+\frac{\sigma_{z}^{2}}{k_{t z} k_{c z}}-\left(\frac{2}{k_{t x} k_{c x}}-\frac{1}{k_{t z} k_{c z}}\right) \sigma_{x} \sigma_{y}-\frac{\sigma_{y} \sigma_{z}+\sigma_{x} \sigma_{z}}{k_{t z} k_{c z}} \\
+\left(\frac{4}{k_{t x} k_{c x}}-\frac{1}{k_{t z} k_{c z}}\right) \tau_{x y}^{2}+\frac{\tau_{y z}^{2}+\tau_{z x}^{2}}{k_{z x}^{2}} \\
+\left(\frac{1}{k_{t x}}-\frac{1}{k_{c x}}\right)\left(\sigma_{x}+\sigma_{y}\right)+\left(\frac{1}{k_{t z}}-\frac{1}{k_{c z}}\right) \sigma_{z}=1
\end{gathered}
$$

It is obvious that material coefficients in plane of transverse isotropy that precede terms $\sigma_{\mathrm{x}} \sigma_{\mathrm{y}}$ and $\tau_{\mathrm{xy}}$ are not fully independent since they contain not only in-plane tensile and compressive strengths $\mathrm{k}_{\mathrm{tx}}, \mathrm{k}_{\mathrm{cx}}$ but also out-of-transverse isotropy plane tensile and compressive strengths $\mathrm{k}_{\mathrm{tz}}, \mathrm{k}_{\mathrm{cz}}$. Consequently, Eq. (5) can be classified as the tetragonal transversely isotropic Tsai-Wu criterion of initial failure.

\section{CONVEXITY LOSS IN CASE OF HIGH ORTHOTROPY}

Applicability range of Tsai-Wu transversely isotropic criterion (5) to properly describe initiation of failure in some engineering materials that exhibit high orthotropy degree, is bounded by a possible elliptic form loss of the limit surface. In other words, a physically inadmissible degeneration of the single convex and simply connected elliptic limit surface into two concave hyperbolas surfaces occurs. The following inequality bounds the range of applicability for Hill's criterion (see Ottosen and Ristinmaa, 2005; Ganczarski and Skrzypek, 2013):

$\frac{1}{k_{t z} k_{c z}}\left(\frac{4}{k_{t x} k_{c x}}-\frac{1}{k_{t z} k_{c z}}\right)>0$

Substitution of the dimensionless parameter $\mathrm{R}=$ $2\left(\mathrm{k}_{\mathrm{tz}} \mathrm{k}_{\mathrm{cz}} / \mathrm{k}_{\mathrm{tx}} \mathrm{k}_{\mathrm{cx}}\right)-1$ (extension of Hosford and Backofen (1964) parameter), leads to the simplified restriction:

$$
R>-0.5
$$

If the above inequalities (6)-(7) do not hold, elliptic cross sections of the limit surface degenerate to two hyperbolic branches and the loss of convexity occurs. To illustrate this limitation, the failure curves in two planes: the transverse isotropy $\left(\sigma_{\mathrm{x}}, \sigma_{\mathrm{y}}\right)$ :

$\sigma_{x}^{2}-\frac{2 R}{1+R} \sigma_{x} \sigma_{y}+\sigma_{y}^{2}+\left(k_{c x}-k_{t x}\right)\left(\sigma_{x}+\sigma_{y}\right)=k_{t x} k_{c x}$

and the orthotropy plane $\left(\sigma_{\mathrm{x}}, \sigma_{\mathrm{z}}\right)$ :

$$
\begin{gathered}
\sigma_{x}^{2}-\frac{2}{1+R} \sigma_{x} \sigma_{z}+\frac{2}{1+R} \sigma_{z}^{2}+\left(k_{c x}-k_{t x}\right) \sigma_{x} \\
+k_{t x} k_{c x}\left(\frac{1}{k_{t z}}-\frac{1}{k_{c z}}\right) \sigma_{z}=k_{t x} k_{c x}
\end{gathered}
$$

for various R-values, are sketched in Fig. $1 a, b$ respectively. It is observed that when $R$, starting from $R=3$, approaches the limit $\mathrm{R}=-0.5$, the curves change from closed ellipses to two parallel lines in transverse isotropy plane or parabola in orthotropy plane, whereas for $\mathrm{R}<-0.5$, concave hyperbolas appear. In general case of strong orthotropy, when the convexity condition (6) does not hold, the Tsai-Wu criterion (5) becomes useless. This effect is decribed in details for the case of Hill's (1948) orthotropic yield criterion (see Ganczarski and Skrzypek, 2013) from which TsaiWu's criterion obviously inherits all inconvenient features since the structure of quadratic terms of both conditions is analogous.

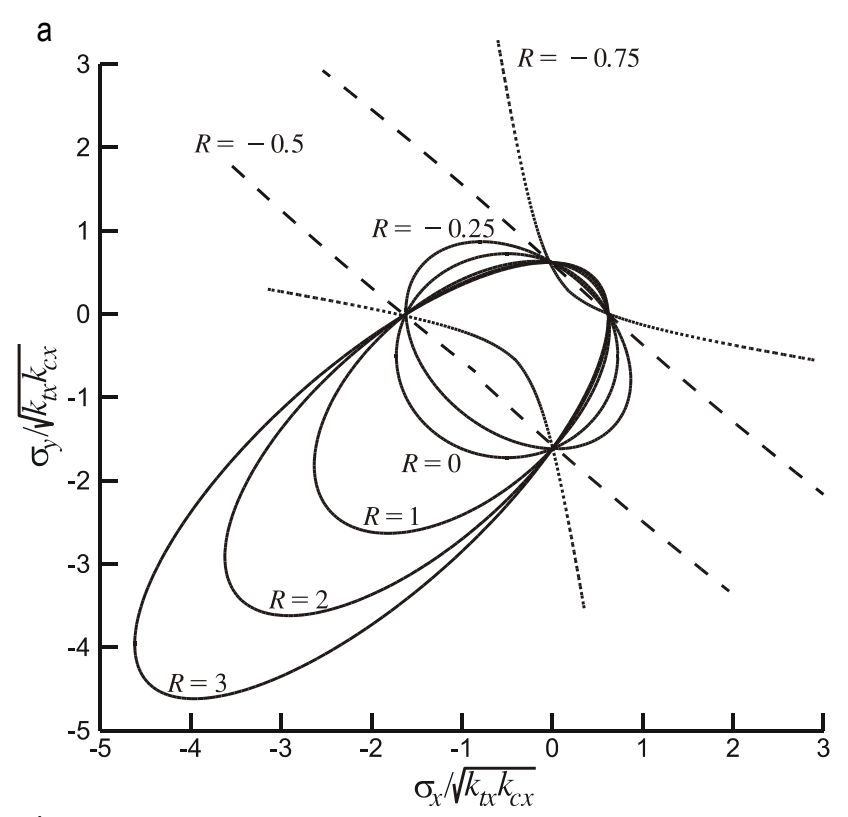

b

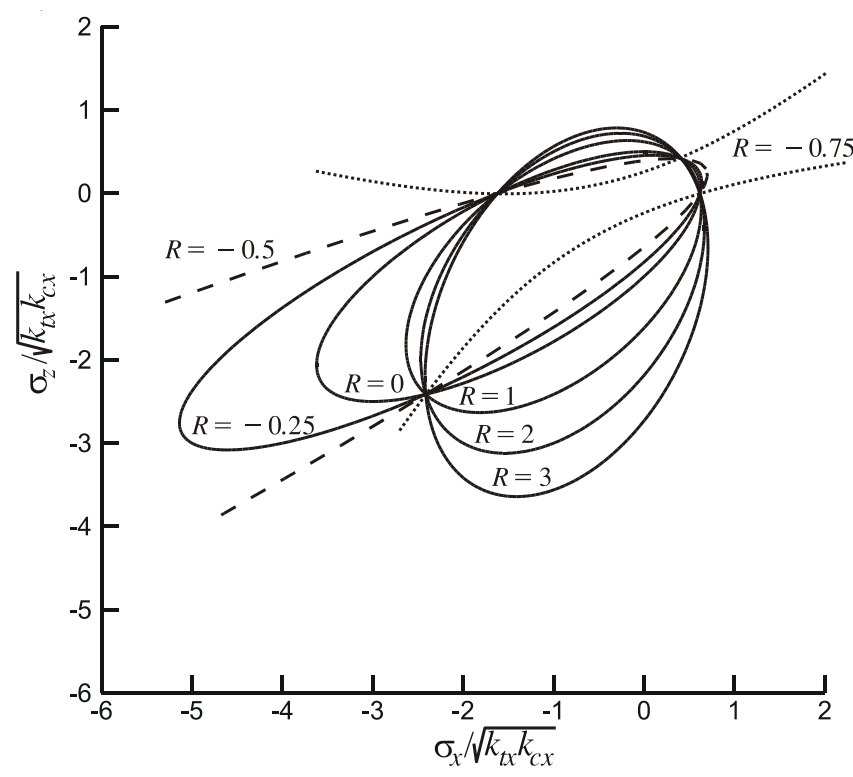

Fig. 1. Degeneration of the Tsai-Wu limit surface with the magnitude of the generalized Hosford and Backhofen parameter $R$ : a) transverse isotropy plane, b) orthotropy plane

\section{MODIFIED TSAI-WU BASED HEXAGONAL FAILURE CRITERION}

Except the tetragonal transversely isotropic Tsai-Wu criterion Eq. (5) one can introduce hexagonally isotropic Tsai-Wu failure criterion:

$$
\begin{gathered}
\frac{\sigma_{x}^{2}-\sigma_{x} \sigma_{y}+\sigma_{y}^{2}}{k_{t x} k_{c x}}+\frac{\sigma_{z}^{2}}{k_{t z} k_{c z}}-\frac{\sigma_{y} \sigma_{z}+\sigma_{x} \sigma_{z}}{k_{t z} k_{c z}}+\frac{3}{k_{t x} k_{c x}} \tau_{x y}^{2}+ \\
\frac{\tau_{y z}^{2}+\tau_{z x}^{2}}{k_{z x}^{2}}+\left(\frac{1}{k_{t x}}-\frac{1}{k_{c x}}\right)\left(\sigma_{x}+\sigma_{y}\right)+\left(\frac{1}{k_{t z}}-\frac{1}{k_{c z}}\right) \sigma_{z}=1
\end{gathered}
$$

in which coefficients that precede terms $\sigma_{\mathrm{x}} \sigma_{\mathrm{y}}$ and $\tau_{\mathrm{xy}}^{2}$ are always positive. These prevent elliptic form of failure curves from degeneration and reduce Eq. (10) to the Huber-von Mises-Hencky ellipse "shifted" outside the origin of co-ordinate system in case of transverse isotropy plane. Since the Huber-von Mises-Hencky 
criterion exhibits isotropy in hexagonal sense, therefore the TsaiWu failure criterion can also be classified as hexagonal in the plane of transverse isotropy. Consequently, condition (10) never violates the Drucker stability postulate, which is not guaranteed by equation (5). However, the hexagonally isotropic Tsai-Wu failure criterion cannot be presented in explicitly deviatoric form (1) which means that the axis of appropriate limit surface in the principal stress space is not parallel to the hydrostatic axis.

\section{RESULTS}

Both the Tsai-Wu transversely isotropic initial failure criteria: tetragonal Eq. (5) and new hexagonal type Eq. (10) are compared for columnar ice in plane of transverse isotropy $\left(\sigma_{x}, \sigma_{y}\right)$ in Fig. 2, in plane of orthotropy $\left(\sigma_{x}, \sigma_{z}\right)$ in Fig. 3 and in plane of transverse isotropy $\left(\sigma_{x}, \tau_{x y}\right)$ in Fig. 4. The experimental data of columnar ice was established by Ralston (1997) in Tab. 1.

Tab. 1. Experimental data for columnar ice, after Ralson (1997)

\begin{tabular}{|c|c|c|c|}
\hline \multicolumn{2}{|c|}{ Tensile strength } & \multicolumn{2}{c|}{ Compressive strength } \\
\hline$k_{t x}$ & $1.01 \mathrm{MPa}$ & $k_{c x}$ & $7.11 \mathrm{MPa}$ \\
\hline$k_{t z}$ & $1.21 \mathrm{MPa}$ & $k_{c z}$ & $13.5 \mathrm{MPa}$ \\
\hline
\end{tabular}

Cross sections of the limit surface are ellipses, that exhibit strong aspect ratio in case tetragonal symmetry, the centers of which are shifted outside the origin of co-ordinate system towards the quarter referring to compressive stresses. In case of cross section by plane of transverse isotropy (see Fig. 2) the symmetry axis has obviously inclination equal $45^{\circ}$ to the axes of coordinate system, in other words it overlaps projection of hydrostatic axis at the transverse isotropy plane $\left(\sigma_{x}, \sigma_{y}\right)$, contrary to the cross section by plane of orthotropy (see Fig. 3 ) the main semi-axis of ellipse is inclined by $71.1^{\circ}$.

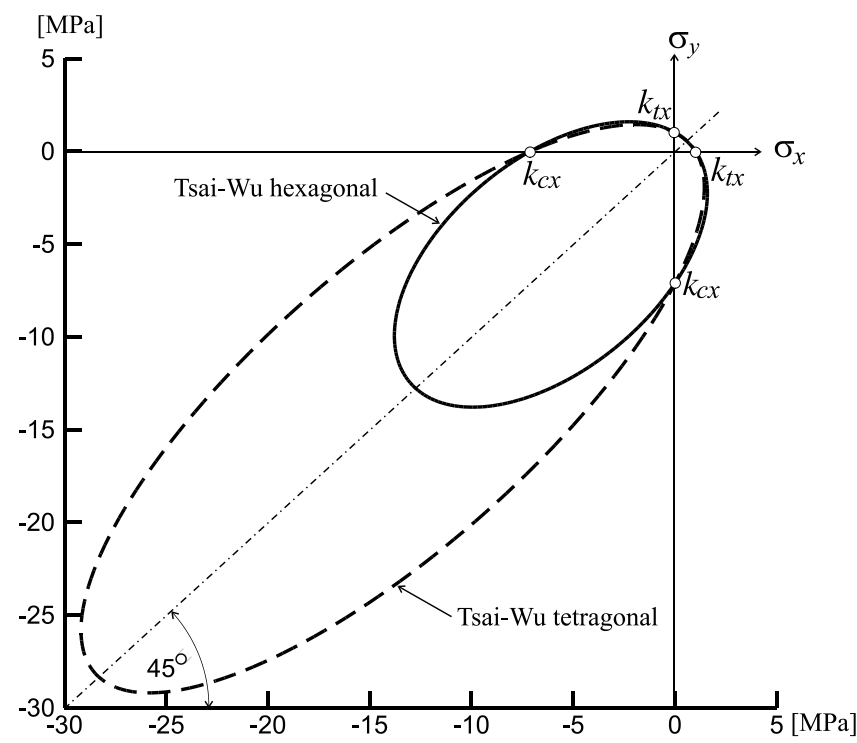

Fig. 2. Comparison of transversely isotropic Tsai-Wu initial failure criteria of tetragonal and hexagonal types for columnar ice in case of plane of transverse isotropy $\left(\sigma_{x}, \sigma_{y}\right)$

It has to be emphasized that, in case of columnar ice, compressive strength along othotropy axis $\mathrm{k}_{\mathrm{cz}}$ is over 10 times great- er than tensile strength $\mathrm{k}_{\mathrm{tz}}$, whereas analogous ratio $\mathrm{k}_{\mathrm{cx}} / \mathrm{k}_{\mathrm{tx}}$ is approximately equal to 7 in case of transverse isotropy plane. Moreover, ratio of semi-axes for Tsai-Wu tetragonal ellipse in $\left(\sigma_{x}, \sigma_{y}\right)$ plane essentially exceeds analogous ratio for Hubervon Mises-Hencky like ellipse, contrary to the case of Tsai-Wu hexagonal ellipse.

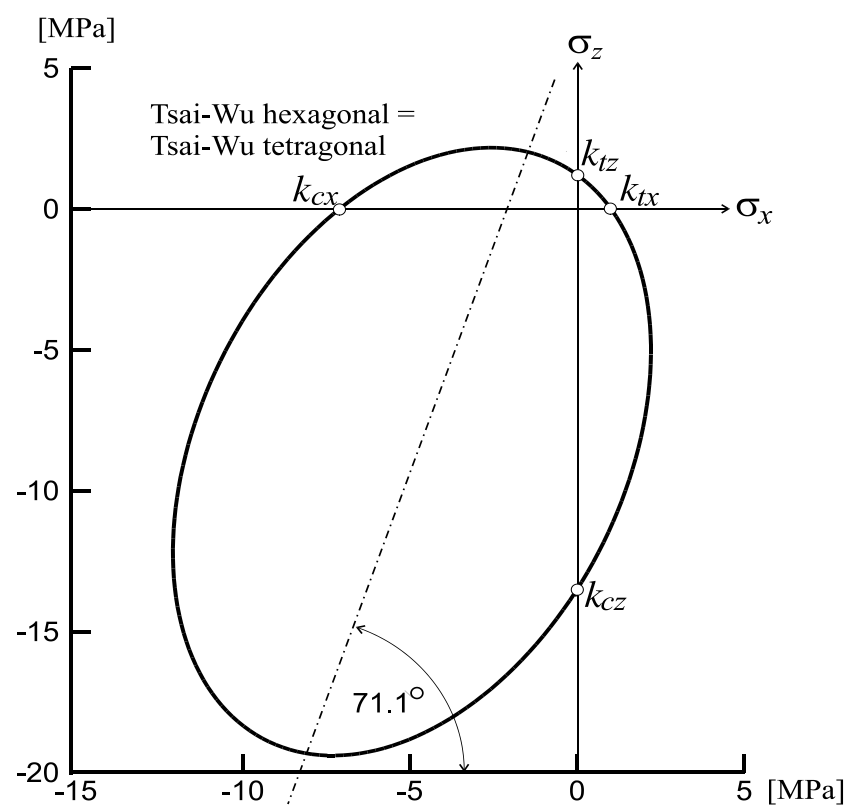

Fig. 3. Comparison of transversely isotropic Tsai-Wu initial failure criteria of tetragonal and hexagonal types for columnar ice in case of plane of orthotropy $\left(\sigma_{x}, \sigma_{z}\right)$

In case of cross sections of the limit surface in shear plane $\left(\sigma_{x}, \tau_{x y}\right)$ both ellipses referring to tetragonal and to hexagonal symmetry have comparable aspect ratio along tensioncompression direction mainly resulting from aforementioned strength difference ratio $\mathrm{k}_{\mathrm{cx}} / \mathrm{k}_{\mathrm{tx}}$ however the criterion (10) performs ellipse slightly broder than the criterion (5).

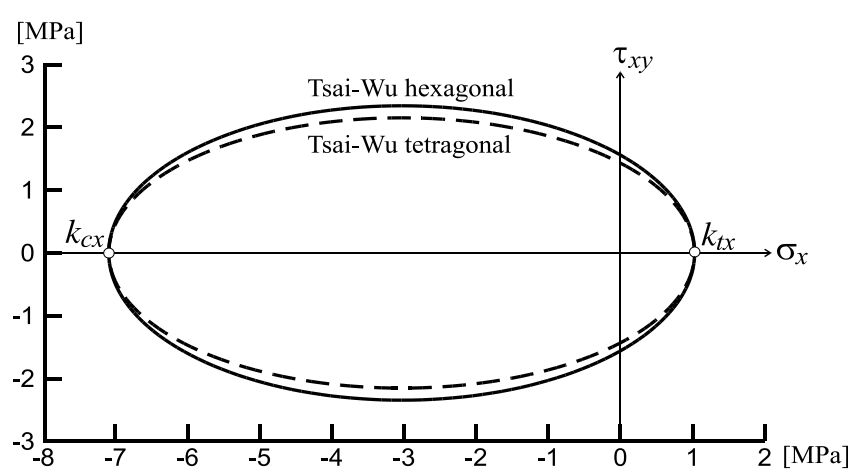

Fig. 4. Transverse isotropic Tsai-Wu initial failure criteria of tetragonal and hexagonal types for columnar ice in case of plane of transverse isotropy $\left(\sigma_{x}, \tau_{x y}\right)$

It is also worth to emphasize that although the tetragonal transversely isotropic Tsai-Wu failure criterion Eq. (5) and the hexagonal transversely isotropic Tsai-Wu failure criterion Eq. (10) contain the same number of 5 independent strengths $\mathrm{k}_{\mathrm{tx}}, \mathrm{k}_{\mathrm{cx}}$, $\mathrm{k}_{\mathrm{tz}}, \mathrm{k}_{\mathrm{cz}}$ and $\mathrm{k}_{\mathrm{zx}}$, only criterion (10) is free from convexity loss and simultaneously truly transversely isotropic in sense of hexagonal class of symmetry. 


\section{CONCLUSIONS}

Both transversely isotropic failure criteria: Tsai-Wu tetragonal and Tsai-Wu hexagonal perform paraboloidal surfaces in the space of principal stresses. However, the tetragonal criterion is represented by elliptic paraboloid which axis is parallel to the hydrostatic axis contrary to the hexagonal one, which represents elliptic paraboloid which axis is not parallel to the hydrostatic axis. Hence, hexagonal Tsai-Wu failure criterion does not satisfy deviatoric property; this is a consequence of its coincidence with Hubervon Mises-Hencky like criterion in the plane of transverse isotropy as well as a property of saving elliptical nature despite of high ratio of orthotropy. Choice of appropriate transversely yield criterion either tetragonal or hexagonal depends on coincidence with experimental tests done on real materials, that can be subjected to one or other class of symmetry, but also can exhibit properties different than aforementioned cases. Key point for proper classification of real transversely isotropic material to one of symmetry class (tetragonal, hexagonal) is the shape of limit curve belonging to the plane of transverse isotropy.

\section{REFERENCES}

1. Cazacu O., Barlat F. (2004), A criterion for description of anisotropy and yield differential effects in pressure-insensitive materials, International Journal of Plasticity, 20, 2027-2045.

2. Chen W.F., Han D.J. (1995), Plasticity for Structural Engineeres, Springer Verlag, Berlin-Heidelberg.

3. Ganczarski A., Skrzypek J. (2013), Mechanics of novel materials, Wydawnictwo Politechniki Krakowskiej (in Polish).

4. Ganczarski A., Skrzypek J. (2014), Constraints on the applicability range of Hill's criterion: strong orthotropy or transverse isotropy, Acta Mechanica, 225, 2563-2582.

5. Goldenblat I. I. Kopnov, V. A. (1966): A generalized theory of plastic flow of anisotropic metals, Stroitielnaya Mekhanika, 307-319, (in Russian).

6. Hill R. (1948), A theory of the yielding and plastic flow of anisotropic metals, Proceedings of Royal Society, London, A193, 281-297.

7. Hosford W. F., Backofen W. A. (1964), Strength and plasticity of textured metals, in W. A. Backhofen, J. Burke, L. Coffin, N. Reed and V. Weisse (eds), Fundamentals of deformation processing, Syracuse University Press, 259-298.

8. Khan A. S., Kazmi R., Farrokh B. (2007), Multiaxial and nonproportional loading responses, anisotropy and modeling of Ti-6Al-4V titanium alloy over wide ranges of strain rates and temperatures, International Journal of Plasticity, 23, 931-950.

9. Murakami S. (2012), Continuum Damage Mechanics, Springer Verlag, Berlin.

10. Ottosen N. S., Ristinmaa M. (2005), The mechanics of constitutive modeling. Elsevier, Amsterdam.

11. Ralson T.D. (1997), Yield and plastic deformation of ice crushing failure, ICSLAIDJEX Symposium on Sea Ice-Processes and Models, Seattle, Washington.

12. Sayir M. (1970), On yield condition in theory of plasticity, Ingenieurarchiv, 39, 414-432 (in German).

13. Tsai S. T., Wu E. M. (1971), A general theory of strength for anisotropic materials, Journal of Composite Materials, 5, 58-80.

The work was supported by National Science Centre Poland grant Nr UMO-2011/03/B/ST8/05132. 